\title{
A COMPARISON OF METHODS FOR THE ESTIMATION OF SERUM CHOLESTEROL AND VALUES IN RANDOM SAMPLES OF POPULATIONS IN THE 55-64 AGE GROUP
}

\author{
BY \\ T. G. MORRIS \\ From the Pneumoconiosis Research Unit, Llandough Hospital, Penarth, Glamorgan
}

(RECEIVED FOR PUBLICATION MARCH 3, 1959)

The increasing mortality from coronary heart disease has focused attention on its epidemiological and biological aspects, and in the latter connexion much work has been done on the relationship between blood cholesterol levels and heart disease. As a result many methods for the estimation of cholesterol levels in serum have been published and their multiplicity indicates the difficulties of the estimation. Reviews published by Zak and Ressler (1955) and Cook (1958) deal with the isolation and estimation, but few comparisons of more than two methods are available.

The estimation has usually to be done on small amounts of material so that semi-micro colorimetric methods are normally used. The majority of these are based upon the LiebermannBurchard reaction. Liebermann described the colour changes which occur when concentrated sulphuric acid is added to a solution of cholesterol in acetic anhydride, and Burchard later developed this by applying the reaction in a nonaqueous solvent such as chloroform. The more sensitive method of Zlatkis, Zak, and Boyle (1953) makes use of the colour developed by cholesterol with concentrated sulphuric acid and ferric chloride. Several modifications of this method have been published, which suggests that it has some technical difficulties.

\section{The Methods}

This paper gives the results of an experiment comparing several methods in current use for the determination of serum cholesterol. The methods chosen were as follows:

(1) The method of Pearson, Stern, and McGavack (1953) was used as published. In it, $p$-toluenesulphonic acid and acetic anhydride are added to the serum, followed later by concentrated sulphuric acid. The colour develops rapidly.
(2) The method of Zlatkis et al. (1953) was also used as published. In it, a mixture of glacial acetic acid, ferric chloride, and concentrated sulphuric acid is added to the serum and the colour measured after a short interval. The results obtained by this method, although consistent among themselves, were very much higher than all the others so that a further investigation was done to examine the effect of purifying the acetic acid used, by the method of Henly (1957).

(3) In this, the method of Trinder (1952), the cholesterol esters in the serum are decomposed by a treatment with alcoholic potash and then all the cholesterol in solution is extracted with petroleum ether. After evaporation of this solvent the cholesterol is dissolved in $1: 2$ dichloroethane and a colour developed by the addition of a mixture of acetyl chloride and concentrated sulphuric acid.

In the original method as published the colour was measured on an absorptiometer with a filter giving a fairly wide waveband of light. The instrument used in the trial was a "unicam" S.P. 600 in which the wavelength of light can be chosen more accurately. Preliminary work showed that the maximum absorption of light occurred at $490 \mathrm{~m} \mu$, so this wavelength was used throughout.

(4) Sackett's (1925) method is a modification of Bloor's (1916) method. The serum is treated with a mixture of alcohol and ether to precipitate proteins and extract the cholesterol. The dissolved cholesterol is recovered by evaporation and then re-dissolved in chloroform to which is added a mixture of acetic anhydride and concentrated sulphuric acid to develop a colour.

The method used here was essentially the same except that time and temperature were standardized, since these factors are critical. Preliminary trials showed that at $25^{\circ} \mathrm{C}$. the colour developed rapidly to a maximum and faded almost as rapidly, as is shown in Fig. 1. Strict time-keeping is therefore necessary and it is essential to time all operations so that readings are taken exactly 15 minutes after the addition of the colour reagent. The effect of varying 


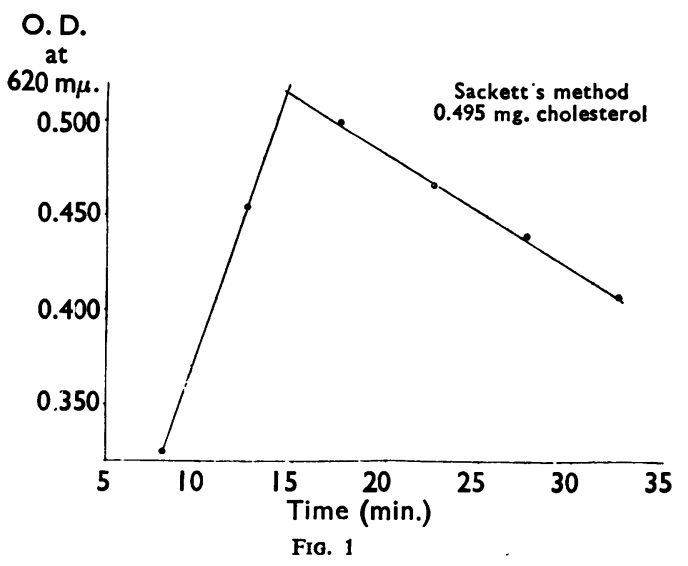

temperatures was not investigated closely, but $25^{\circ} \mathrm{C}$. was chosen arbitrarily, since it is high enough to be above laboratory temperature and therefore a thermostat can be used, and low enough to make cooling unnecessary before measuring the colour.

(5) The method of Abell, Levy, Brodie, and Kendall (1952), as modified by Anderson and Keys (1956), is the K.5 modification which has been used by Keys on his surveys. The serum is treated with alcoholic potash and the cholesterol then extracted with petroleum ether. After evaporation, the dry residue of cholesterol is dissolved in a mixture of acetic anhydride, sulphuric acid, and acetic acid to develop the colour. To overcome the fading difficulties, readings of the colour are taken at intervals of time and the maximum colour developed thus obtained by extrapolation.

(6) The method of Schoenheimer and Sperry (1934), as modified by Sperry and Webb (1950), was used. In this the serum is treated with alcohol and acetone and after filtration of the protein floc the cholesterol in the filtrate is precipitated as the digitonide by the addition of digitonin solution.

The precipitate is separated, dissolved in hot glacial acetic acid, and then the colour developed by the addition of an acetic-anhydride-sulphuric acid mixture. Strict time-keeping is again necessary, but only one reading of the colour is taken.

\section{Test Samples}

The sera used were chosen on the basis of preliminary tests so that the cholesterol content was spread over as wide a range as possible. Since it was impossible to do all the determinations in one day, the samples had to be stored. It is generally assumed that when serum is frozen the esterified cholesterol level may change but the total cholesterol level remains unaffected, although little experimental evidence seems to be available. A method of determination of total cholesterol which does not decompose esters may therefore give different results in a fresh serum from those on the same serum after freezing. Storing at $4^{\circ} \mathrm{C}$. does not affect the esterified cholesterol. Two methods of storage were used in this test. In the first enough of the sample for a determination in triplicate, by any one method, was placed in a bijou bottle and kept in deep freeze for at least 30 days to stabilize any changes due to freezing. In the other method of storage a bulk sample of each serum was kept in the refrigerator at $4^{\circ} \mathrm{C}$. and aliquots measured out as required.

\section{Standard Cholesterol Solution}

The results given by all the methods are based upon a solution of cholesterol of known strength using cholesterol of known purity, which in these tests was B.D.H. cholesterol recrystallized from absolute alcohol and dried to constant weight over phosphorus pentoxide in a partial vacuum. The melting point of the final product was $148.5-149.5^{\circ} \mathrm{C}$. Aliquots of this solution were pipetted our and evaporated to dryness in suitable tubes. The solid residue of cholesterol was then dissolved in measured amounts of whichever solvent the particular method in use required.

\section{Samples from Randomly Selected Populations}

Groups of males and females between the ages of 55 and 64 selected at random from the population in the Vale of Glamorgan have been studied by Thomas, Cochrane, and Higgins (1958) and in the course of this study blood samples were taken. The samples of blood were drawn from the arm vein and no attempt was made to take the samples at definite intervals after meals. The patients were invited to attend the clinic and whenever they arrived the sample was drawn, so that it could have been taken at any time from 9 a.m. to 9 p.m. After sampling, the blood was allowed to clot naturally, ringed, and kept in a refrigerator overnight, transported to the laboratory, and the serum separated. Blood samples were also obtained under similar conditions from males of the same age group in a survey in Annandale carried out by Kennedy and Cowan (1958). In both surveys over $90 \%$ of the patients in the sample chosen were actually examined.

\section{Results}

The results obtained by the different methods on the samples used are given in Table $I$. Unfortunately the results by the method of Pearson et al. on Samples 1 to 5 were lost.

A statistical analysis has been done on the results after the figures obtained by the methods of Pearson et al. and Zlatkis et al. have been excluded since these were obviously much higher than any of the others.

The standard error of a single determination by Trinder's, Keys and others' (K.5), and Sperry and Webb's methods did not differ significantly, but the error of Sackett's method is significantly higher. The mean value obtained by Sackett's method is significantly higher than that of the 
TABLE I

CHOLESTEROL LEVELS (MG. \%) IN SERUM OBTAINED BY DIFFERENT METHODS

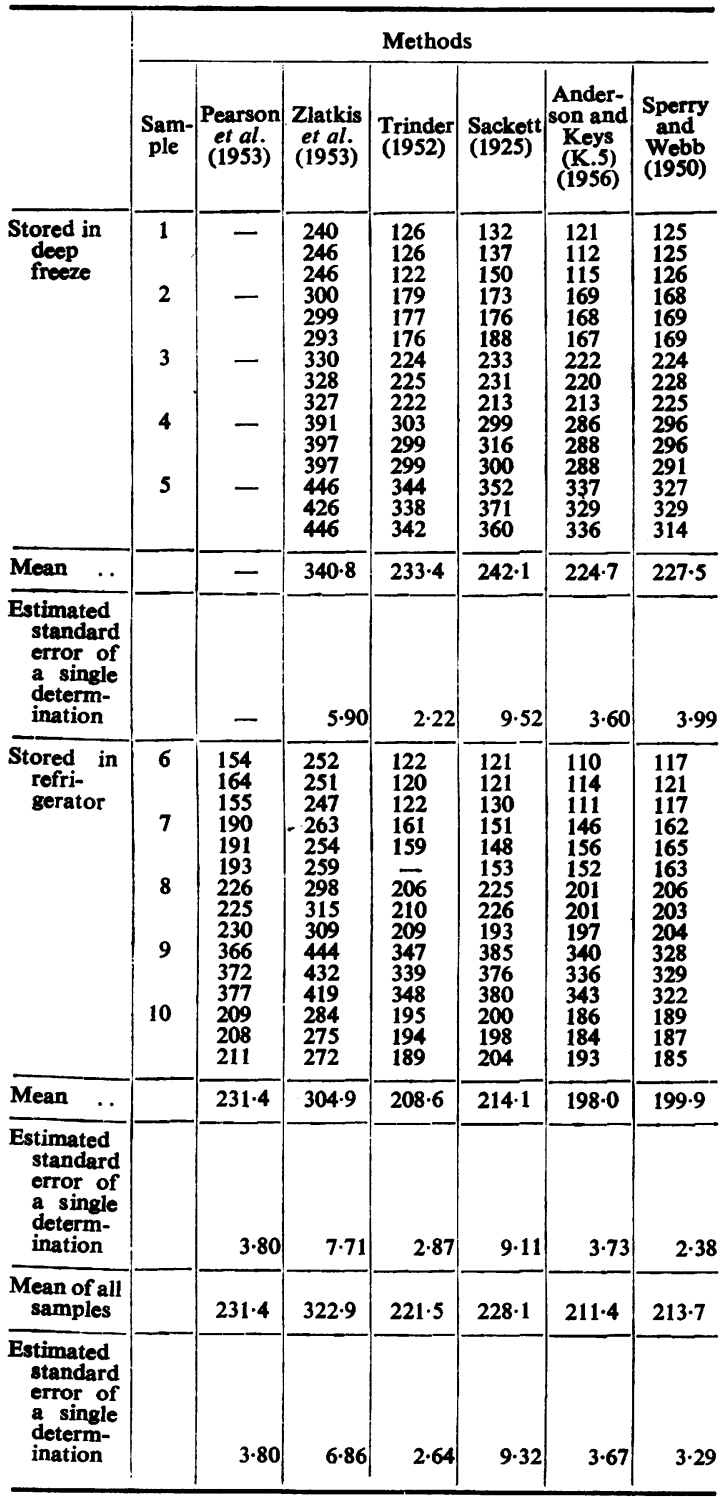

other three methods, and the mean by Trinder's method is significantly higher than that of Anderson and Keys (K.5) and Sperry and Webb's methods, which agree.

As mentioned earlier, the high results obtained by the method of Zlatkis et al. prompted a further study of the effect of purifying the acetic acid, which entails refluxing the acetic acid with chromium trioxide. Results with treated acid and the same acid before treatment are given in Table II.

The results obtained with treated acid are considerably lower than those with untreated, but the mean values are still significantly higher than all the other methods. The standard error of a single determination was reduced by acid treatment and did not differ from that of Trinder's, Anderson and Keys's (K.5), and Sperry and Webb's methods found previously.

It is apparent from the current literature that results obtained by this method usually agree better with those of other methods than they did in this experiment. This experience seems to indicate the need for considerable caution in using the technique as published.

\section{Random Sample of Population}

Cholesterol levels were determined by Trinder's method and the results are given in Figs. 2 and 3.

\section{Discussion}

Results of Analyses by Different Methods.The methods chosen can be divided into two types on the basis of technique. In the methods of Pearson $e t$ al. and Zlatkis et al. the reagents are added directly to the serum and in the others the cholesterol is extracted, before estimation, either

TABLE II

CHOLESTEROL LEVELS (MG. \%) IN SERUM ESTIMATED BY METHOD OF ZLATKIS ET AL. (1953)

\begin{tabular}{|c|c|c|}
\hline Sample & Untreated Acid & Treated Acid \\
\hline $\begin{array}{l}1 \\
2 \\
3 \\
4 \\
4 \\
5 * \\
6 \\
7 \\
8 \\
9 \\
10\end{array}$ & $\begin{array}{l}272 \\
281 \\
276 \\
336 \\
324 \\
338 \\
380 \\
374 \\
367 \\
446 \\
457 \\
457 \\
305 \\
302 \\
308 \\
296 \\
307 \\
302 \\
358 \\
360 \\
358 \\
531 \\
526 \\
531 \\
380 \\
365 \\
361\end{array}$ & $\begin{array}{l}161 \\
162 \\
161 \\
219 \\
215 \\
215 \\
270 \\
270 \\
272 \\
347 \\
351 \\
356 \\
169 \\
170 \\
171 \\
208 \\
208 \\
208 \\
267 \\
266 \\
266 \\
411 \\
403 \\
406 \\
266 \\
273 \\
270\end{array}$ \\
\hline Mean .. & $366 \cdot 6$ & $257 \cdot 8$ \\
\hline $\begin{array}{l}\text { Estimated S.E. of } \\
\text { single observa- } \\
\text { tion } . . \quad \ldots\end{array}$ & 5.86 & 1.96 \\
\hline
\end{tabular}

* There was no sample available for a repeat determination. 


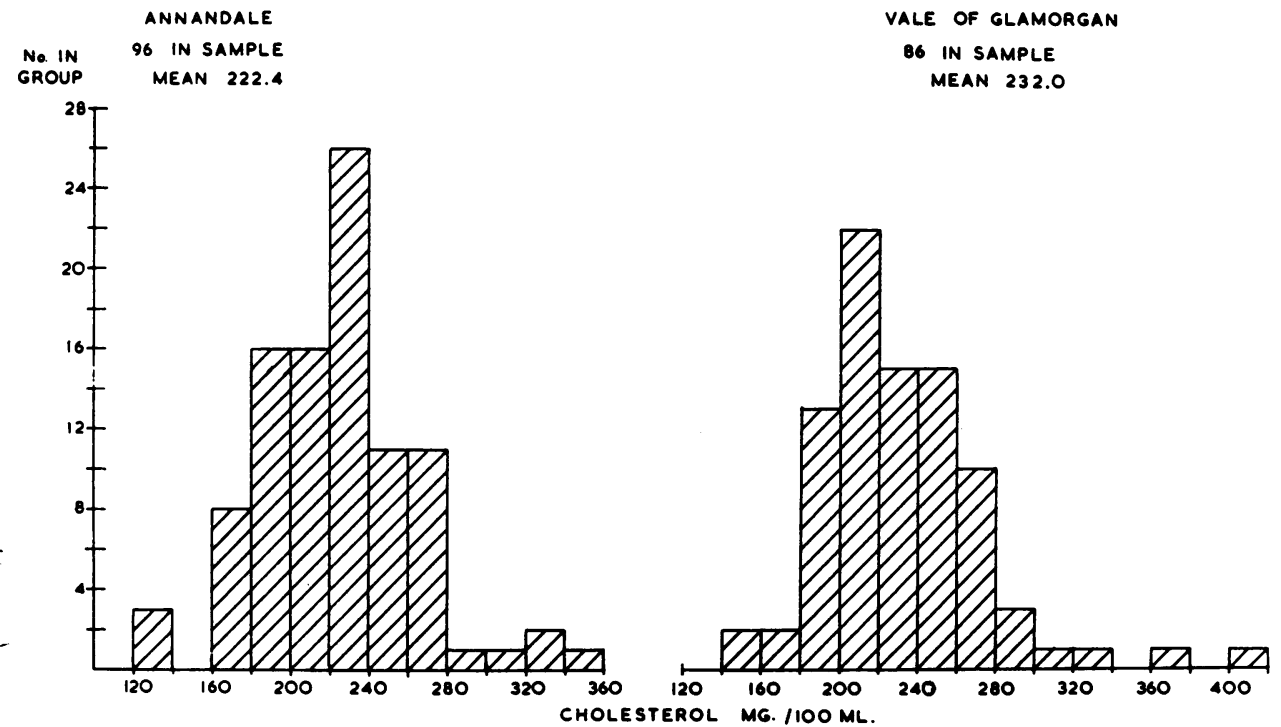

FIG. 2.-Total serum cholesterol in males aged 55-64 years.

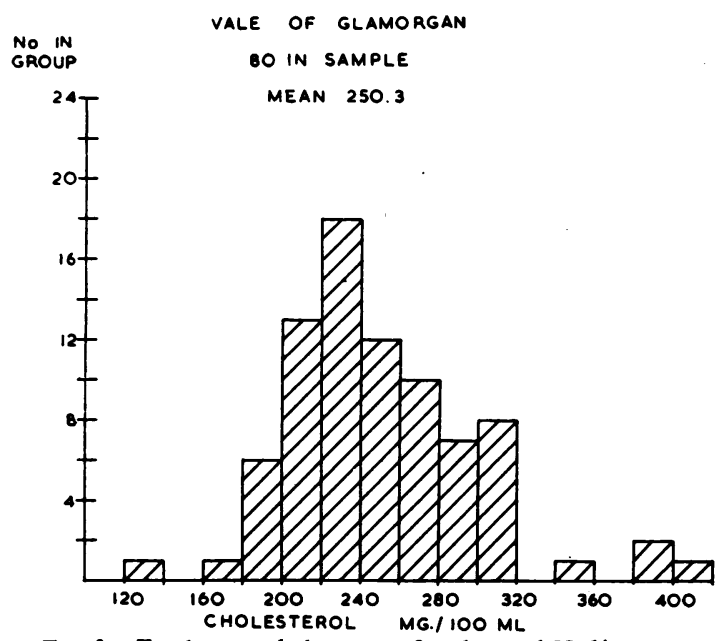

FiG. 3.-Total serum cholesterol in females aged 55-64 years.

by solvents or by digitonin precipitation. The results show that the methods in which the reagents are added to the serum are less accurate than the others. Since all sera are coloured, some strongly, the use of these methods necessitates the setting up of blanks for each sample and this cuts down the number of samples that can be done. It is not clear, also, that other substances in the serum are inert to the colour reagents, and in fact a recent modification of the method of Zlatkis et al. does extract the cholesterol from the serum (Herrmann, 1957). In this method the sulphuric acid-ferric chloride reagent is very viscous and the final mixture of glacial acetic acid and the reagent is also viscous, which means that transferring the solution from the tubes to colorimeter cells is difficult if air bubbles are to be avoided. Also washing the cells out after measuring the colour is tedious. This latter is, of course, a small point, but in a series of determinations using a timetable such small points can assume importance.

In the methods of Sackett, of Trinder, and of Anderson and Keys the cholesterol is extracted by organic solvents and in the Sperry and Webb method by precipitation with digitonin. Of the organic solvent methods, in that of Sackett a mixed alcohol-ethyl ether solvent is employed and, in the other two, petroleum ether. Sackett's method is the least satisfactory in that two transfers of the cholesterol are required, which doubles the chance of loss, and also a time-consuming centrifugation. is necessary.

In the petroleum ether methods, the extraction follows a treatment with alcoholic potash designed to saponify any esters of cholesterol. The method of Anderson and Keys (K.5) employs two separate extractions while that of Trinder one only. From the results it appears that one is quite as efficient as two, since the values by Trinder's method are the same as those with Anderson and Keys's.

The colour is developed in the methods of both Sackett and Anderson and Keys by a sulphuricacid-acetic-anhydride mixture. It has to be prepared freshly each day, as do all the colour reagents in the methods used, but the conditions of preparation involve cooling in an ice-bath, which is not always convenient. 
In Trinder's method the reagent is a mixture of acetyl chloride and sulphuric acid. It can be prepared at room temperature and used at that temperature. It reacts violently with water, but under normal conditions of the method there is no water present. It fumes slightly in air and it is convenient to add the reagent in a fume chamber but it is not essential. The reagent is no more unpleasant than the acetic-anhydride-sulphuricacid reagent.

The green colour in the methods of Sackett and Anderson and Keys is labile, and in Sackett's method much care has to be taken to read the intensity of colour at exactly 15 minutes after addition of the reagent. In the method of Anderson and Keys readings are taken at intervals and the peak intensity determined. In Trinder's method the red colour develops in 15 minutes and is then stable for at least an hour although careful time-keeping is advisable.

The digitonin method of Sperry and Webb is not usually used for routine work, since it involves an overnight wait for precipitation of the digitonide. The pre-treatment using alcoholacetone is liable to give trouble in that the mixture on the steam bath can "bump" badly with loss even from narrow-necked flasks.

When considering methods in the foregoing manner it is very difficult to avoid bias. In this laboratory the Trinder method has been adopted and many hundreds of determinations have been done. As a result, other methods tend to appear tedious and awkward. This may be due to real difficulties in the techniques or it may be due to the fact that, for the small amount of work necessary for the comparison, the techniques have not been used enough to become familiar and therefore easier.

It is of interest here that in the Johns Hopkins School of Medicine, Thomas and Eisenberg (1957), using an adapted Sackett method, obtained standard errors on separate occasions of 11.2 and $10.1 \mathrm{mg}$. cholesterol per $100 \mathrm{ml}$., which are similar to the 9.3 obtained here. They also quote figures by other workers using the method of Abell et al. of 7.6 to $14.5 \mathrm{mg}$. per $100 \mathrm{ml}$., depending on the workers. Anderson and Keys's modification of the method of Abell et al. here gave a standard error of $3.7 \mathrm{mg}$. per $100 \mathrm{ml}$., which is considerably better.

It would appear, however, that the best methods for use on a routine basis are either those of Anderson and Keys or Trinder, and the latter is quicker, since serial readings of colour are not taken. From considerable experience of the method and comparisons of results obtained by workers using the (K.5) modification of Anderson and Keys with those on the same material by Trinder's method, the difference was thought to be less than the $4 \%$ found here. In Trinder's original paper his own comparisons with the Sperry and Webb method are closer than $2 \%$.

It is not possible to compare the two methods of storage on the cholesterol level since samples of the same sera were not kept under both conditions. The standard error of a single determination by any method does not appear to be affected by the type of storage.

Results of Determinations on the Random Samples.-The populations from which the random samples were drawn were, in both cases, predominantly agricultural, although there was, of course, a proportion of professional and industrial workers and some retired persons. The particular age group of 55-64 does not appear to have been studied as such to any great extent, but several workers have studied populations with age groups contained in, or containing, this group.

Adlersberg, Schaefer, Steinberg, and Chun-I Wang (1956), using the Schoenheimer digitonin method, examined a group of males and females, chiefly of Italian, Irish, English, and Scottish origin, in New York, and in the age group 58-62 found a mean cholesterol level among the males of 236.2 and females of $263.8 \mathrm{mg}$. per $100 \mathrm{ml}$. In the present survey in this age group the mean cholesterol level in the Vale of Glamorgan was 240.2 for the males and $259.1 \mathrm{mg}$. per $100 \mathrm{ml}$. in the females, while in Annandale the level for males was $223.9 \mathrm{mg}$. per $100 \mathrm{ml}$, which is quite good agreement.

Epstein, Boas, and Simpson (1957) studied a group composed almost entirely of Italians and Jews, so that their results are probably less comparable than those of Adlersberg et al. with the present series, but they did find that, regardless of origin, the mean cholesterol level (using the method of Abell et al.) of the females was higher than that of the males. In the present study the mean cholesterol level of the males of both groups did not differ significantly, but that of the females in the Vale was significantly higher.

Within the age group studied, although the numbers are small, a breakdown into separate years showed a general upward trend in mean cholesterol level over the years 55-59 and then a fall to the age of 64 , which appears to be fairly consistent with the findings of both Adlersberg et al. and Epstein et al.

\section{Summary}

Increasing mortality from coronary disease has focused attention on the cholesterol level of blood 
as one of the many factors connected with the aetiology of the disease.

Many methods have been proposed for the difficult estimation of blood cholesterol levels, and six of these methods, namely, those of Pearson et al. (1953), Zlatkis et al. (1953), Trinder (1952), Sackett (1925), Anderson and Keys (K.5) (1956), and Sperry and Webb (1950), have been compared by carrying out determinations in triplicate by each method on 10 sera.

The method of Trinder was the most reproducible, with those of Anderson and Keys (K.5) and Sperry and Webb the next most reproducible. Sackett's method was the least reproducible.

Mean levels obtained with the methods of Sperry and Webb and Anderson and Keys (K.5) did not differ ; the mean by Trinder's method was $4.2 \%$ higher and the mean by Sackett's method $7.3 \%$ higher than these two. The methods of Pearson et al. and Zlatkis et al. gave means $8.8 \%$ and $51.8 \%$ higher respectively.

The methods of Trinder and Anderson and Keys (K.5) are the most suitable for routine use, and Trinder's method involves less manipulation and is quicker.

Cholesterol levels in random samples of populations in different areas, in the age group
55-64, were determined by Trinder's method to gain basic information for comparative purposes.

The author wishes to place on record his thanks to Dr. J. C. Gilson for his help and encouragement and to Drs. A. L. Cochrane and A. C. Kennedy for their help in organizing the samples. Especial thanks are due to Mr. P. D. Oldham for his keen interest and for his invaluable assistance in planning the experiment and to Mr. Peter Cross for analysing the results.

\section{REFERENCES}

Abell, L. L., Levy, B. B., Brodie, B. B., and Kendall, F. E. (1952), J. biol. Chem., 195, 357.

Adlersberg, D., Schaefer, L. E., Steinberg, A. G., and Chun-I Wang (1956). J. Amer. med. Ass., 162, 619.

Anderson, J. T., and Keys, A. (1956). Clin. Chem., 2, 145.

Bloor, W. R. (1916). J. biol. Chem., $24,227$.

Cook, R. P., Ed. (1958). Cholesterol. Academic Press, New York. Epstein, F. H., Boas, E. P., and Simpson, R. (1957). J. chron. Dis., 5, 300 .

Henly, A. A. (1957). Analyst, 82, 286.

Herrmann, R. G. (1957). Proc. Soc. exp. Biol. (N.Y.), 94, 503.

Kennedy, A. C., and Cowan, B. (1958). Scot. med. J., 3, 283.

Pearson, S., Stern, S., and McGavack, T. H. (1953). Analyt. Chem., 25, 813 .

Sackett, G. E. (1925). J. biol. Chem., 64, 203.

Schoenheimer, R., and Sperry, W. M. (1934). Ibid., 106, 745.

Sperry, W. M., and Webb, M. (1950). Ibid., 187, 97.

Thomas, A. J., Cochrane, A. L., and Higgins, I. T. T. (1958). Lancet, 2,540 .

Thomas, C. B., and Eisenberg, F. F. (1957). J. chron. Dis., 6, 1.

Trinder, P. (1952). Analyst, 77, 321.

Zak, B., and Ressler, N. (1955). Amer. J. clin. Path., 25, 433.

Zlatkis, A., Zak, B., and Boyle, A. J. (1953). J. Lab. clin. Med., 41, 